\title{
Treatment of severely ill COVID-19 patients with anti-interleukin drugs (COV-AID): A structured summary of a study protocol for a randomised controlled trial
}

Bastiaan Maes $^{*+} \mathbb{D}$, Cedric Bosteels ${ }^{\dagger}$, Elisabeth De Leeuw ${ }^{\dagger}$, Jozefien Declercq $^{\dagger}$, Karel Van Damme $^{\dagger}$, Anja Delporte, Bénédicte Demeyere, Stéfanie Vermeersch, Marnik Vuylsteke, Joren Willaert, Laura Bollé, Yuri Vanbiervliet, Jana Decuypere, Frederick Libeer, Stefaan Vandecasteele, Isabelle Peene and Bart Lambrecht

\begin{abstract}
Objectives: The purpose of this study is to test the safety and effectiveness of individually or simultaneously blocking IL-6, IL-6 receptor and IL-1 versus standard of care on blood oxygenation and systemic cytokine release syndrome in patients with COVID-19 coronavirus infection and acute hypoxic respiratory failure and systemic cytokine release syndrome.

Trial design: A phase 3 prospective, multi-center, interventional, open label, 6-arm 2x2 factorial design study.

Participants: Subjects will be recruited at the specialized COVID-19 wards and/or ICUs at 16 Belgian participating hospitals. Only adult ( $\geq 18 y$ old) patients will be recruited with recent ( $\leq 16$ days) COVID-19 infection and acute hypoxia (defined as $\mathrm{PaO} 2 / \mathrm{FiO} 2$ below $350 \mathrm{mmHg}$ or $\mathrm{PaO} 2 / \mathrm{FiO} 2$ below 280 on supplemental oxygen and immediately requiring high flow oxygen device or mechanical ventilation) and signs of systemic cytokine release syndrome characterized by high serum ferritin, or high D-dimers, or high LDH or deep lymphopenia or a combination of those, who have not been on mechanical ventilation for more than 24 hours before randomisation. Patients should have had a chest X-ray and/or CT scan showing bilateral infiltrates within the last 2 days before randomisation. Patients with active bacterial or fungal infection will be excluded.

Intervention and comparator: Patients will be randomized to 1 of 5 experimental arms versus usual care. The experimental arms consist of Anakinra alone (anti-IL-1 binding the IL-1 receptor), Siltuximab alone (anti-IL-6 chimeric antibody), a combination of Siltuximab and Anakinra, Tocilizumab alone (humanised anti-IL-6 receptor antibody) or a combination of Anakinra with Tocilizumab in addition to standard care. Patients treated with Anakinra will receive a daily subcutaneous injection of $100 \mathrm{mg}$ for a maximum of 28 days or until hospital discharge, whichever comes first. Siltuximab $(11 \mathrm{mg} / \mathrm{kg})$ or Tocilizumab $(8 \mathrm{mg} / \mathrm{kg}$, with a maximum dose of $800 \mathrm{mg})$ are administered as a single intravenous injection immediately after randomization.

(Continued on next page)
\end{abstract}

* Correspondence: bastiaan.maes@irc.vib-ugent.be

${ }^{\dagger}$ Bastiaan Maes, Cedric Bosteels, Elisabeth De Leeuw, Jozefien Declercq and Karel Van Damme are joint first authors.

VIB-UGent Inflammatie-researchcentrum, Oost-Vlaanderen, Ghent, Belgium

(c) The Author(s). 2020 Open Access This article is licensed under a Creative Commons Attribution 4.0 International License, which permits use, sharing, adaptation, distribution and reproduction in any medium or format, as long as you give appropriate credit to the original author(s) and the source, provide a link to the Creative Commons licence, and indicate if changes were made. The images or other third party material in this article are included in the article's Creative Commons licence, unless indicated otherwise in a credit line to the material. If material is not included in the article's Creative Commons licence and your intended use is not permitted by statutory regulation or exceeds the permitted use, you will need to obtain permission directly from the copyright holder. To view a copy of this licence, visit http://creativecommons.org/licenses/by/4.0/ The Creative Commons Public Domain Dedication waiver (http://creativecommons.org/publicdomain/zero/1.0/) applies to the data made available in this article, unless otherwise stated in a credit line to the data. 
(Continued from previous page)

Main outcomes: The primary end point is the time to clinical improvement defined as the time from randomization to either an improvement of two points on a six-category ordinal scale measured daily till day 28 or discharge from the hospital or death. This ordinal scale is composed of (1) Death; (2) Hospitalized, on invasive mechanical ventilation or ECMO; (3) Hospitalized, on non-invasive ventilation or high flow oxygen devices; (4) Hospitalized, requiring supplemental oxygen; (5) Hospitalized, not requiring supplemental oxygen; (6) Not hospitalized.

Randomisation: Patients will be randomized using an Interactive Web Response System (REDCap). A 2x2 factorial design was selected with a 2:1 randomization regarding the IL-1 blockade (Anakinra) and a 1:2 randomization regarding the IL-6 blockade (Siltuximab and Tocilizumab).

Blinding (masking): In this open-label trial neither participants, caregivers, nor those assessing the outcomes are blinded to group assignment.

Numbers to be randomised (sample size): A total of 342 participants will be enrolled: 76 patients will receive usual care, 76 patients will receive Siltuximab alone, 76 patients will receive Tocilizumab alone, 38 will receive Anakinra alone, 38 patients will receive Anakinra and Siltuximab and 38 patients will receive Anakinra and Tocilizumab.

Trial Status: COV-AID protocol version 3.0 (15 Apr 2020). Participant recruitment is ongoing and started on April $4^{\text {th }}$ 2020. Given the current decline of the COVID-19 pandemic in Belgium, it is difficult to anticipate the rate of participant recruitment.

Trial registration: The trial was registered on Clinical Trials.gov on April 1st, 2020 (ClinicalTrials.gov Identifier: NCT04330638) and on EudraCT on April 3rd 2020 (Identifier: 2020-001500-41).

Full protocol: The full protocol is attached as an additional file, accessible from the Trials website (Additional file 1). In the interest in expediting dissemination of this material, the familiar formatting has been eliminated; this Letter serves as a summary of the key elements of the full protocol.

Keywords: COVID-19, Randomised controlled trial, protocol, systemic cytokine release syndrome, hypoxic respiratory failure, interleukin 6 blockade, interleukin 1 blockade, Anakinra, Siltuximab, Tocilizumab, Acute Respiratory Distress Syndrome

\section{Supplementary information}

Supplementary information accompanies this paper at https://doi.org/10. 1186/s13063-020-04453-5.

Additional file 1. Full study protocol.

Acknowledgements

Not applicable.

\section{Authors' contributions}

$B M, C B, K V D, E D L$ and JD equally contributed to the study and its submission. BL, CB, BM, KVD, EDL, JD and SVdc, screened patients for eligibility and obtained informed consents. BL, CB, BM, KVD, EDL and SVdC were involved in data collection, analysis and interpretation of the data. MV was involved in the statistical considerations for the study design. AD, BD and SV coordinated data collection and administration across the different sites involved. JW, LB, YV, JD and FL were involved in the data input. IP and $B L$ wrote the protocol. The author(s) read and approved the final manuscript.

\section{Funding}

The Belgian Health Care Knowledge Centre (KCE, Federaal Kenniscentrum voor de Gezondheidszorg) funds the COV-AID trial and is involved in the trial design, data analysis and interpretation and publication.
Ethics approval and consent to participate

This study was approved by an independent Medical Ethics Committee attached to the University Hospital of Ghent and the University of Ghent (reference BC-07562) on April 3rd 2020, after consultation of the ethics committees of each Belgian centre where this study will be carried out. Eligible subjects may only be included in the study after providing written Ethics Committee-approved informed consent, or, if incapable of doing so, after such consent has been provided by a legally acceptable representative(s) of the subject, after receiving full explanation, having received sufficient time to considerer the trial, asking questions and receiving satisfying responses to all questions

Consent for publication

Not applicable.

\section{Competing interests}

The authors declare that they have no competing interests.

Received: 24 May 2020 Accepted: 24 May 2020

Published online: 03 June 2020

\section{Publisher's Note}

Springer Nature remains neutral with regard to jurisdictional claims in published maps and institutional affiliations. 